\title{
Evidence of a Heterogeneous Nucleus in a Polypropylene Spherulite Using Electron Probe Microanalysis
}

\author{
Kiyoshi Ikeda and Genzo Hashizume \\ Industrial Research Institute of Hyogo Prefecture, \\ 3, Yukihira-cho, Suma-ku, Kobe, Japan. \\ (Received February 6, 1975)
}

\begin{abstract}
The behavior of nucleating agents in the crystallization of polypropylene (PP) was investigated by an electron probe microanalyzer (EPMA). Aluminum benzoate (AlB) and sodium benzoate $(\mathrm{NaB})$ were used as the nucleating agents. In a PP spherulite containing $\mathrm{NaB}$, sodium $\mathrm{K}_{\alpha}$ was detected in the neighborhood of the spherulitic center. This result indicates that $\mathrm{NaB}$ granules act as nuclei for PP spherulites. It is also confirmed that the AlB granule exists at the spherulitic center.
\end{abstract}

KEY WORDS Polypropylene / Spherulite / Nucleating Agent / Electron Probe Microanalyzer /

Heterogeneous nuclei in polypropylene and several other polymers considerably change the crystallization kinetics, morphology and physical properties of the base polymers. ${ }^{1-3}$ Various Materials, such as catalyst residues, ${ }^{4}$ organocarboxylic acid salts ${ }^{5}$ and inorganic compounds ${ }^{6}$ have been reported as heterogeneous nucleating agents for polymer crystallization. In most cases the function of the nucleating agent was assessed by measuring its effect on the polymer supercooling ${ }^{5,7}$ and the crystallization mechanism of the PP-nucleating agent compound was explained using the modified Avrami equation ${ }^{8}$ on the assumption that nucleating agents act as heterogeneous nuclei for polymer crystallization.

However, few papers have been published on a microscopic description of the compounds. The present study was undertaken to elucidate the behavior of $\mathrm{AlB}$ and $\mathrm{NaB}$ granules in PP spherulites during the crystallization process by comparison of EPMA data with those of thermal analysis.

\section{EXPERIMENTAL}

\section{Materials and Preparation}

Noblene w 101 produced by Sumitomo Chemical Co. Ltd. $\left(M_{v}=7 \times 10^{4}\right)$ was used as base PP without further purification. AlB and $\mathrm{NaB}$ were used as nucleating agents for PP because both chemicals were reported as good nucleating agents, ${ }^{5,8}$ and both chemicals were reagent grade commercial materials used without further purification. $\mathrm{AlB}$ and $\mathrm{NaB}$ were passed through a sieve of 325 mesh to obtain fine granules. A proper amount of the granules was vigorously stirred with $\mathrm{PP}$ in xylene at about $130^{\circ} \mathrm{C}$ until the granules were homogeneously dispersed. After being dried to remove xylene, the AlB - and NaB-PP compounds were pressed at $200^{\circ} \mathrm{C}$ to a film of about $20 \mu \mathrm{m}$ in thickness.

In order to make the morphological difference between the AlB - or NaB-PP compound and the base PP visible, the film sandwiched between glass slides, was isothermally crystallized for $1 \mathrm{hr}$ at $150 \pm 3^{\circ} \mathrm{C}$ after being melted for $10 \mathrm{~min}$ at $220^{\circ} \mathrm{C}$, and was then quenched in ice-water solution. Films of AlB-PP, NaB-PP and base $\mathrm{PP}$, were observed using a polarizing microscope. For scanning electron microscopy (SEM), the films stripped from the glass slides were etched in a solution containing 20-\% sulfuric acid saturated with chromium trioxide for $5 \mathrm{~min}$ at $70^{\circ} \mathrm{C} .^{9,10}$

\section{DSC and EPMA Measurements}

After being maintained for $10 \mathrm{~min}$ at $220^{\circ} \mathrm{C}$ to exclude thermal history, the peak crystallization temperatures of the compounds were measured on a Perkin-Elmer DSC $1 \mathrm{~B}$ with a constant cooling rate of $10^{\circ} \mathrm{C} / \mathrm{min}$.

EPMA measurements were carried out with a 
Shimazu EPMA type EMX-SM. The etched film was made electroconductive with evaporated carbon and an analyzing crystal of rubidium acid phthalate was used to detect aluminum $\mathrm{K} \alpha$ and sodium $\mathrm{K} \alpha$.

\section{RESULTS AND DISCUSSION}

When AlB or $\mathrm{NaB}$ is blended with PP, the peak crystallization temperature of the compound depends on the concentration of AlB or NaB. Plots of the temperature of each compound against the concentration of $\mathrm{AlB}$ and $\mathrm{NaB}$ are shown in Figure 1. The peak temperature becomes higher with increasing concentration of each agent. The increase in peak temperature due to AlB and $\mathrm{NaB}$ is smaller than that indicated in the literature. ${ }^{7,8}$ The difference in peak crystallization temperature between the literature and this experiment seems to depend on the difference in degree of granule dispersion.

Nevertheless, from the fact that the peak temperature obviously differs from that of the original polymer at high concentrations of AlB or $\mathrm{NaB}$, it is evident that both $\mathrm{AlB}$ and $\mathrm{NaB}$ play an important role in the crystallization process of PP.

In order to obtain a clearer picture of the relation between the PP spherulite and AlB or $\mathrm{NaB}$, the compounds containing 1-wt $\% \mathrm{NaB}$ and 0.5 -wt $\%$ AlB were observed through a polarizing microscope and an EPMA. Polarizing

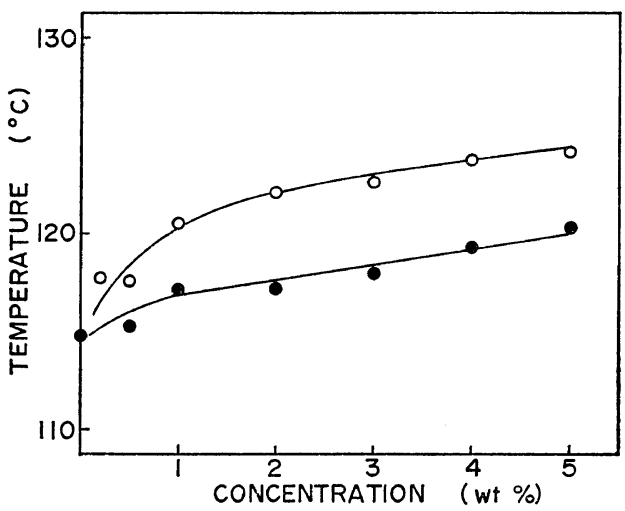

Figure 1. Temperature of maximum crystallization rate of polypropylene with a constant cooling rate of $10^{\circ} \mathrm{C} / \mathrm{min}$ for nucleating agents; aluminum benzoate $(\mathrm{O})$, sodium benzoate $(\mathbf{O})$.

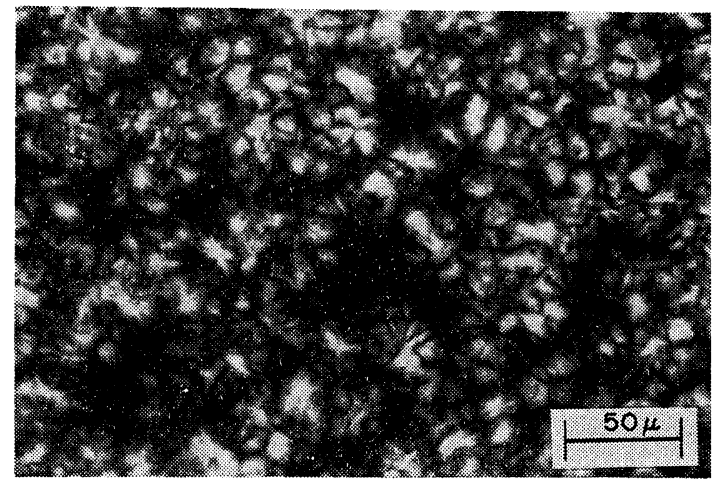

(a)

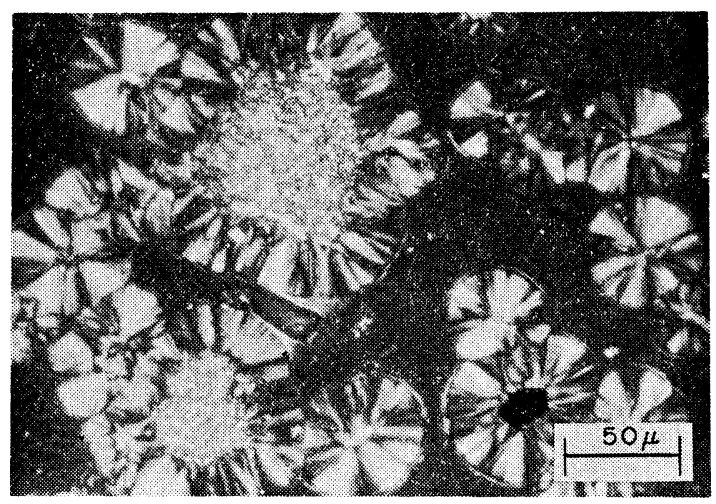

(b)

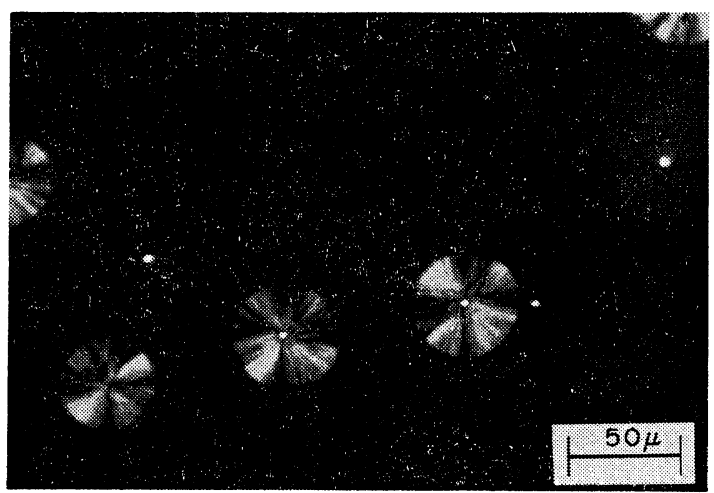

(c)

Figure 2. Polarizing microphotographs of polypropylene spherulites crystallized for $1 \mathrm{hr}$ at $150^{\circ} \mathrm{C}$; (a) AlB-PP compound, (b) NaB-PP compound, (c) base PP.

microphotographs of AlB-PP, NaB-PP and base PP under the same crystallization conditions $\left(1 \mathrm{hr}\right.$ at $\left.150^{\circ} \mathrm{C}\right)$ are shown in Figures $2 \mathrm{a}, \mathrm{b}$, and c, respectively. Few spherulites appear in the 


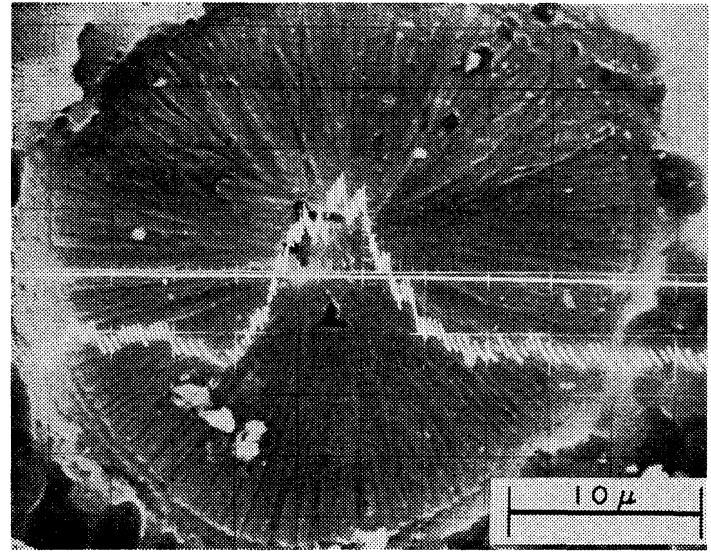

Figure 3. Photograph of polypropylene spherulite containing 1-wt $\% \mathrm{NaB}$ taken under the following conditions: accelerating voltage, $15 \mathrm{kV}$; specimen current, $0.2 \mathrm{nA}$. Line analysis was done with respect to sodium $\mathrm{K} \alpha$ with a specimen current of $0.005 \mu \mathrm{A}$.

base PP but there are a number of small size spherulites in the A1B-PP compound and spherulites which have heterogeneities at the spherulitic centers develop in the $\mathrm{NaB}-\mathrm{PP}$ compound. Figure 3 shows a SEM of a PP spherulite containing 1-wt $\% \mathrm{NaB}$. Line analysis of sodium $\mathrm{K} \alpha$ along the horizontal line of the photograph indicates the presence of $\mathrm{NaB}$ in the neighborhood of spherulitic center. The central area of this SEM is shown at a higher magnification in Figure $4 \mathrm{a}$ and compared with the scanning display of sodium $\mathrm{K} \alpha$ on the same field as shown in Figure 4b. Comparison of the SEM with the scanning display of sodium $\mathrm{K} \alpha$ indicates that sodium distributes like a dull ring at the central region of the spherulite. It was considered that the sodium-poor position at the center of the ringed distribution was a vestige from which a part of the $\mathrm{NaB}$ granule had been eluted in the etching procedure. Other sodiumrich positions correspond to the white particles. These white particles are $\mathrm{NaB}$ granules which are made to move from the original positions by the etching treatment and left behind on the surface of the spherulite.

In the case of a compound containing 0.5wt $\% \mathrm{AlB}$, the granules of AlB exists likewise at the spherulitic center, as shown in Figure 5, where a large size spherulite obtained with slow

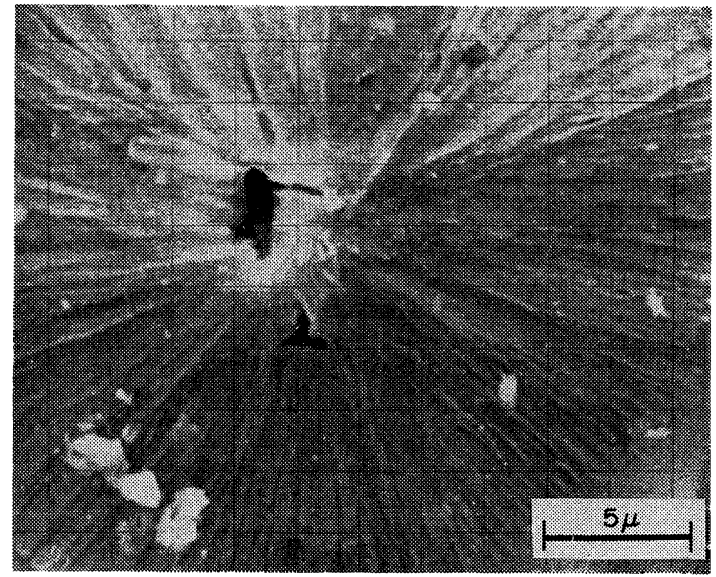

(a)

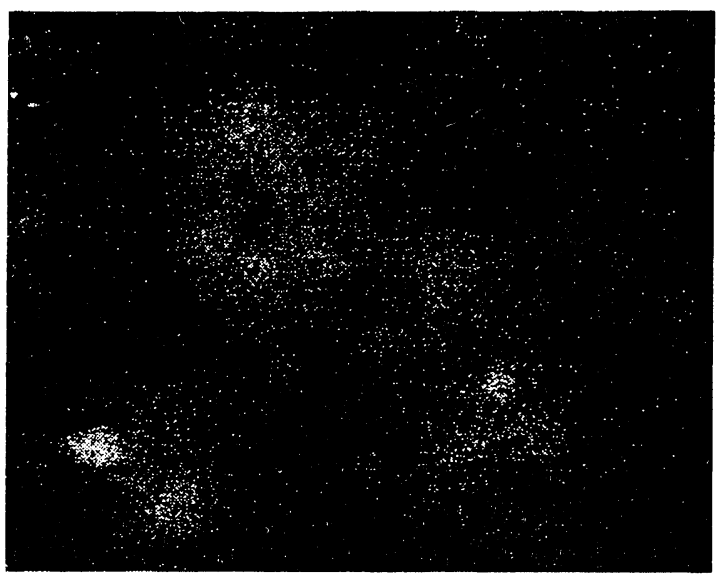

(b)

Figure 4. Comparison of (a) scanning electron micrograph with (b) scanning display of sodium $\mathrm{K} \alpha$ : accelerating voltage, $15 \mathrm{kV}$ (a), (b); specimen currents, $0.2 \mathrm{nA}$ (a), $0.005 \mu \mathrm{A}$ (b).

cooling to room temperature was observed because a number of small size spherulite appear under the same crystallization condition as $\mathrm{NaB}-\mathrm{PP}$.

Furthermore, on the central regions of many spherulites, vestiges in which $\mathrm{NaB}$ was considered to exist were observed in some fields of the scanning electron microscope. In adeition, the crystalline region was distinguished from the noncrystalline region in a film of few $\mu \mathrm{m}$ in thickness when the transmission electron was detected employing a higher accelerating valtage, and sodium was confirmed by $\mathrm{Na} \mathrm{K} \alpha$. 
Evidence of a Heterogeneous Nucleus in a PP Spherulite

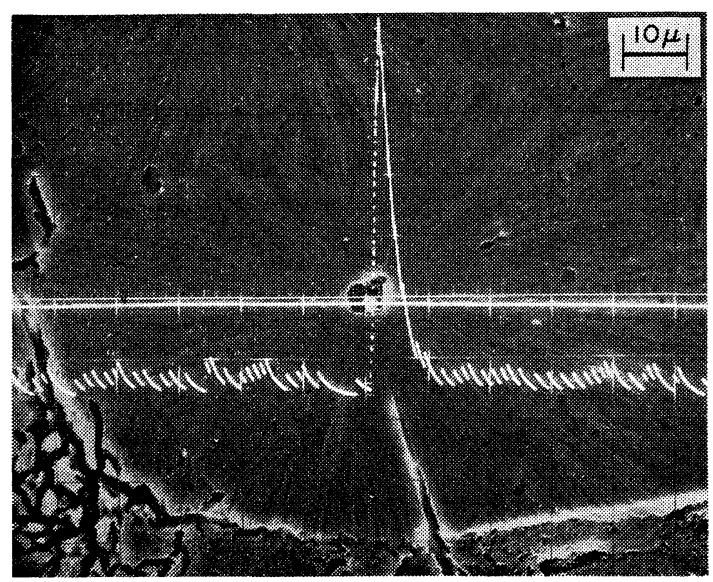

Figure 5. Photograph of polypropylene spherulite containing $0.5-w t \%$ AlB. Accelerating voltage and specimen current were the same as employed in Figure 3.

\section{CONCLUSION}

In the PP compound containing $\mathrm{AlB}$ or $\mathrm{NaB}$ granules in which it was ascertained by thermal analysis that their presence had changed the peak crystallization temperature, AlB and NaB granules were confirmed to exist at the spherulitic center by an EPMA.

It is therefore concluded that $\mathrm{AlB}$ and $\mathrm{NaB}$ granules act as heterogeneous nuclei for the PP spherulites. On the basis of thermal analysis these results give further evidence of the concept that $\mathrm{AlB}$ and $\mathrm{NaB}$ granules are heterogeneous nuclei for PP crystallization.

Acknowledgment. The authors wish to thank T. Yokoyama for EPMA measurement, and M. Motoyama for helpful discussion on several points in the paper.

\section{REFERENCES}

1. F. L. Binsbergen and B. G. De Lange, Polymer, 11, 309 (1970).

2. M. Inoue, J. Polym. Sci., Part A, 1, 2013 (1963).

3. H. N. Beck and H. D. Ledbetter, J. Appl. Polym. Sci., 9, 2131 (1965).

4. J. Boon, G. Halla, and D. W. Van Krevelen, J. Polym. Sci., Part A-2, 6, 1835 (1968).

5. H. N. Beck, J. Appl. Polym. Sci., 11, 673 (1967).

6. G. Groeninckx, H. Berghmans, N. Overbergh, and G. Smets, J. Polym. Sci., Part A-2, 12, 303 (1974).

7. Von U. Johnsen, G. Spilgies, and H. G. Zachmann, Kolloid-Z. Z. Polym., 240, 762 (1970).

8. Von U. Johnsen and G. Spilgies, ibid., 250, 1174 (1972).

9. D. R. Fitchmun and Z. Mencik, J. Polym. Sci., Part A-2, 11, 951 (1973).

10. L. Bartosiewicz and Z. Mencik, ibid., Part A-2, 12, 1163 (1974). 